\title{
Differential effects of aging on spatial learning through exploratory navigation and map reading
}

\section{Naohide Yamamoto* and Gregory J. DeGirolamo}

Department of Psychology, Cleveland State University, Cleveland, OH, USA

Edited by:

Thomas Wolbers, University of

Edinburgh, UK

\section{Reviewed by:}

Scott Moffat, Wayne State

University, USA

Jon Kelly, lowa State University, USA

\section{${ }^{*}$ Correspondence:}

Naohide Yamamoto, Department of

Psychology, Cleveland State

University, 2121 Euclid Avenue,

Cleveland, OH 44115, USA

e-mail:n.yamamoto@csuohio.edu
It has been shown that abilities in spatial learning and memory are adversely affected by aging. The present study was conducted to investigate whether increasing age has equal consequences for all types of spatial learning or impacts certain types of spatial learning selectively. Specifically, two major types of spatial learning, exploratory navigation and map reading, were contrasted. By combining a neuroimaging finding that the medial temporal lobe (MTL) is especially important for exploratory navigation and a neurological finding that the MTL is susceptible to age-related atrophy, it was hypothesized that spatial learning through exploratory navigation would exhibit a greater decline in later life than spatial learning through map reading. In an experiment, young and senior participants learned locations of landmarks in virtual environments either by navigating in them in the first-person perspective or by seeing aerial views of the environments. Results showed that senior participants acquired less accurate memories of the layouts of landmarks than young participants when they navigated in the environments, but the two groups did not differ in spatial learning performance when they viewed the environments from the aerial perspective. These results suggest that spatial learning through exploratory navigation is particularly vulnerable to adverse effects of aging, whereas elderly adults may be able to maintain their map reading skills relatively well.

Keywords: aging, spatial learning, navigation, map, ground-level, aerial, route, survey

\section{INTRODUCTION}

Abilities in spatial learning and memory are adversely affected by both normal and pathological aging. Many senior citizens experience great difficulty in navigation in unfamiliar environments (Burns, 1999), and it is often the case that topographical disorientation is among the earliest symptoms displayed by patients with Alzheimer's disease (Pai and Jacobs, 2004). Results from experimental studies have been consistent with these epidemiological observations, showing that elderly participants (either healthy or demented) performed worse than young participants in a variety of spatial learning and memory tasks (for review, see Iachini et al., 2009; Moffat, 2009; Vlček, 2011). In spite of these converging and accumulating findings in the literature, psychological mechanisms of this age-related decline are poorly understood. For example, it is largely unknown whether aging has equal consequences for all types of spatial learning or impacts certain types of spatial learning selectively. The objective of the present study was to address this issue by contrasting the effects of normal aging on spatial memories acquired through two major methods of spatial learning: exploratory navigation in a ground-level (also called route) perspective and map reading in an aerial (also called survey) perspective (Siegel and White, 1975; Thorndyke and Hayes-Roth, 1982).

Previous studies demonstrated age-related decrements of spatial learning both in exploratory navigation and in map reading. Extensive research has been carried out by having participants learn environments through exploratory navigation in the first-person (i.e., ground-level) perspective. Some studies involved real navigation in physical environments (e.g., Kirasic, 1991; Barrash, 1994; Wilkniss et al., 1997; Newman and Kaszniak, 2000; Iachini et al., 2005), and others utilized simulated navigation in virtual environments (e.g., Moffat et al., 2001; Moffat and Resnick, 2002; Lövdén et al., 2005; Iaria et al., 2009; Zakzanis et al., 2009; Liu et al., 2011). These studies commonly found that young participants acquired knowledge of the environments more accurately or quickly than senior participants (see also Cushman et al., 2008). Similarly, when participants learned specific routes on maps first and attempted to trace them in actual environments later, older adults were slower and less accurate (Wilkniss et al., 1997; Carelli et al., 2011). In addition, young adults tended to use maps more effectively for spatial learning than elderly adults: young adults recalled more landmark locations than elderly adults after studying street maps (Thomas, 1985; De Beni et al., 2006; Meneghetti et al., 2011), and having a map of an environment during exploratory navigation did not enhance elderly adults' learning of object locations (Sjölinder et al., 2005). All of these findings indicates that certain degrees of decline in spatial learning abilities take place in later life regardless of the ways in which an environment is learned. However, because spatial learning by exploratory navigation and spatial learning by map reading were examined separately in the previous studies, it remains unclear whether these two kinds of spatial learning show the same level of decline or one goes down more significantly than the other. Thus, the 
present study was designed to make a direct comparison between exploratory navigation and map reading to investigate possible differential effects of aging on these two types of spatial learning.

Neuroimaging and neurological studies have provided important clues for addressing this issue. On one hand, neuroimaging studies have suggested that activation in the medial temporal lobe (MTL), hippocampus and parahippocampal cortex in particular, is a key to distinguishing spatial learning through exploratory navigation and spatial learning through map reading. For example, Shelton and colleagues found that encoding of spatial information through exploratory navigation and map reading elicited activations in overlapping areas of the young adult brain, but the MTL was activated more strongly by exploratory navigation than map reading (Shelton and Gabrieli, 2002; Shelton and Pippitt, 2007). When Borghesani et al. (2008) applied the same paradigm to healthy elderly adults who did not carry the apolipoprotein E $\varepsilon 4$ allele, a known genetic risk factor for Alzheimer's disease (Strittmatter et al., 1993), they found that MTL activity during spatial learning through exploratory navigation but not through map reading was correlated with subsequent performance on a spatial memory test. Furthermore, when similar activations in the MTL were observed while participants learned environmental layouts in other studies, they typically learned environments through exploratory navigation (e.g., Aguirre et al., 1996; Grön et al., 2000; Moffat et al., 2006; Antonova et al., 2009; Iglói et al., 2010; Marchette et al., 2011; but see also Blanch et al., 2004). On the other hand, cross-sectional and longitudinal studies of agerelated brain volume changes have revealed significant atrophy of the MTL in healthy elderly populations (Golomb et al., 1993; Jack et al., 1997, 1998; Šimić et al., 1997; Insausti et al., 1998; Jernigan et al., 2001; Scahill et al., 2003; Raz et al., 2004; but see also Sullivan et al., 1995, 2005). Importantly, this shrinkage of the MTL has actual behavioral consequences on spatial learning: Nedelska et al. (2012) demonstrated that smaller volume of the right hippocampus corresponds to larger impairment of spatial learning in the first-person perspective. Similarly, impaired performance of elderly adults in spatial learning through exploratory navigation correlated with reduced activation of the MTL, which might result from the atrophy of this brain region (Meulenbroek et al., 2004; Moffat et al., 2006; Antonova et al., 2009). Together, these neuroimaging and neurological findings lead to a hypothesis that vulnerability of the MTL to age-related atrophy makes spatial learning through exploratory navigation more susceptible to detrimental effects of increasing age. By contrast, because the MTL is engaged to a lesser extent while encoding spatial information from maps, it is also predicted that spatial learning through map reading would be affected by aging less significantly.

These hypotheses were tested in an experiment reported below. Young and senior participants learned locations of landmarks in virtual environments by viewing them either from a perspective of an observer walking through the environments (i.e., exploratory navigation) or from an aerial perspective that had a constant orientation relative to the environments (i.e., map reading). After learning each environment, they attempted to reproduce the layout of landmarks in a smaller scale. It was predicted that senior participants would reconstruct the layouts less accurately than young participants. More specifically, a greater difference between young and senior groups would be observed following spatial learning through exploratory navigation than that through map reading.

\section{MATERIALS AND METHODS 2.1. PARTICIPANTS}

Forty-eight participants gave their informed consent to participate in the experiment. They included 24 young participants (9 males and 15 females, 18-33 years old) and 24 senior participants (13 males and 11 females, 60-80 years old). Means $(M)$ and standard deviations $(S D)$ of their ages were as follows: $M=21, S D=4.76$ (young); and $M=68.5, S D=5.19$ (senior). The exact age of one young participant was not available. These two groups differed significantly in mean ages but not in ratios of males and females, $t_{(45)}=-31.95, p<0.001$ and $\chi_{(1)}^{2}=1.34$, $p=0.25$, respectively. They received either monetary compensation or partial credit in a psychology course in return for their participation. They were native or fluent speakers of English and reported normal or corrected-to-normal vision.

All but one senior participant were enrolled in courses at Cleveland State University at the time of the experiment. Thus, although no formal screening was conducted, it was reasonable to assume that they were cognitively healthy individuals.

\subsection{DESIGN AND PROCEDURE}

The experimental protocol described below was approved by the institutional review board of Cleveland State University. Four virtual environments of the same size $(110 \times 130 \mathrm{ft}$ in virtual space) were presented on the display of a desktop computer by using the PsyScope program (Cohen et al., 1993). They were visually distinct from each other (see Figure 1 for examples), and each environment contained 10 large landmarks and seven small objects in a unique configuration. They were the same environments as those used in previous studies by Shelton and colleagues (e.g., Shelton and Gabrieli, 2002; Shelton and McNamara, 2004; Shelton and Pippitt, 2007). Each participant viewed all four environments, two from the perspective of a six-foot-tall observer walking through the environments (navigation condition; Figure 1A) and the other two from the perspective of an observer who was $70 \mathrm{ft}$ above the ground and looking straight down (map condition; Figure 1B). In both conditions, approximately 2-3 landmarks were visible in their entirety at any given time (see Figures $\mathbf{1 A}$ and $\mathbf{B}$ ). The walkthrough of an environment in the navigation condition always began at the southwest corner and proceeded clockwise along the perimeter of the environment (Figure 1C). At each corner, the observer made a $90^{\circ}$-turn to face the new direction of travel. Viewing of an environment in the map condition was carried out in a similar manner. The observer was initially at the southwest corner and moved along four legs of the environment in the same direction. An important difference is that in the map condition the observer maintained the initial orientation (indicated by the arrow labeled as Leg 1 in Figure 1C) within each environment. Variable orientations and the fixed orientation were utilized in navigation and map conditions, respectively, 
A Navigation condition

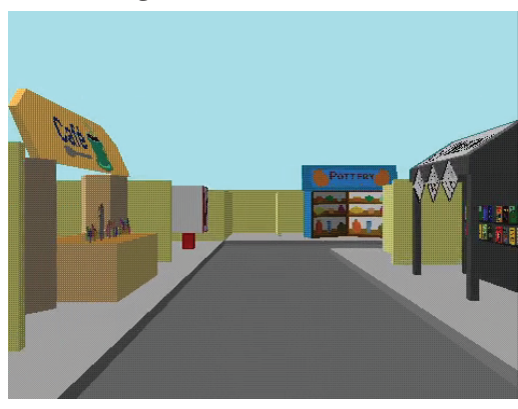

B Map condition

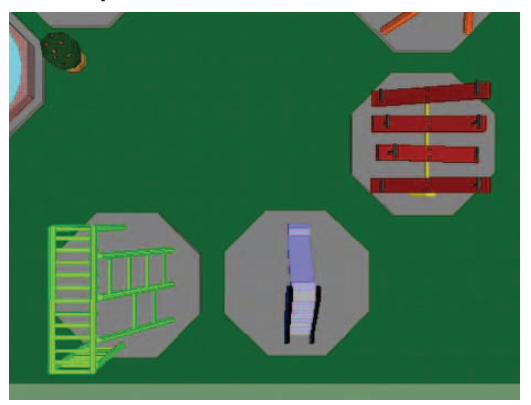

c The overall shape of an environment

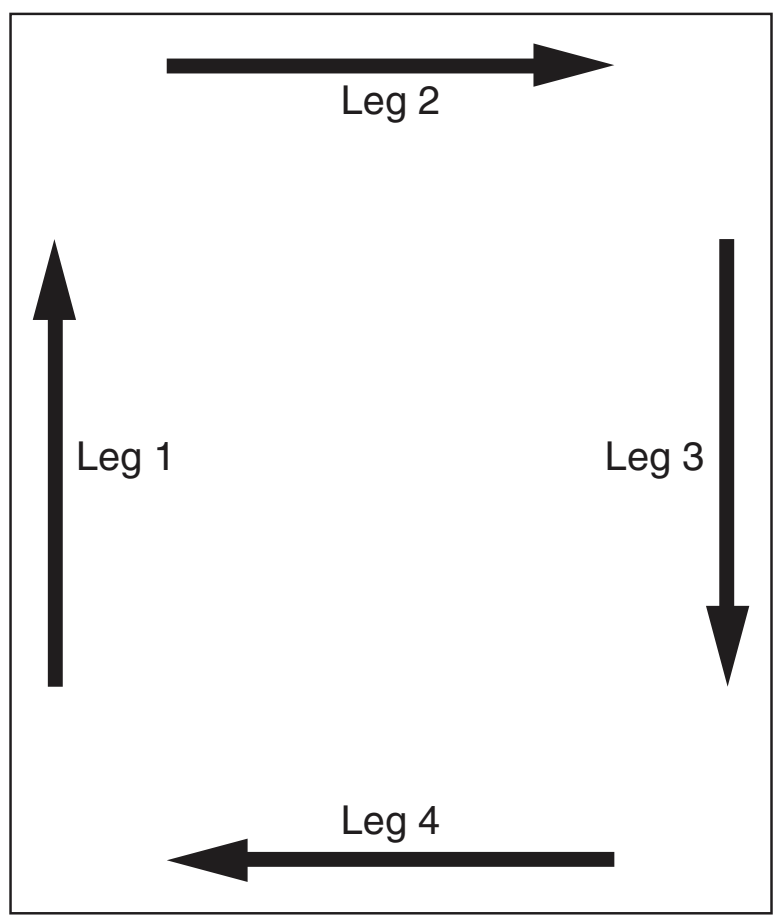

FIGURE 1 | Examples of virtual environments learned by participants in the experiment. They were presented either from a ground-level perspective in varying orientations (A) or from an aerial perspective in a fixed orientation (B). The presentation of each environment began at its southwest corner (lower left corner in the figure) and proceeded clockwise along the four legs (C). These virtual environments were originally created and used by Shelton and colleagues (Shelton and Gabrieli, 2002; Shelton and McNamara, 2004). in order to capture essential aspects of corresponding types of spatial learning: exploratory navigation is conducted in the firstperson perspective that varies with an observer, whereas maps are typically read in a single orientation (Shelton and Pippitt, 2007).

Participants were instructed to learn the locations of the 10 landmarks for a later memory test. They were given a diagram similar to Figure 1C and informed that they would view environments along the four legs in clockwise direction. They were also told that environments would be presented from either a ground-level or an aerial perspective. During the first run of each environment, an experimenter named 10 landmarks that were to be learned. Subsequently, participants viewed six additional runs of the environment by themselves. They were encouraged to pay close attention to all of the subsequent showings of the environment by naming the landmarks every time they appeared on the screen. To clearly indicate which leg they were in at any given moment, a label specifying a leg number was presented for $4 \mathrm{~s}$ right before each leg was shown. Each run of an environment took $56 \mathrm{~s}$ including the leg labels.

After learning each environment, participants were presented with a dry-erase board on which a rectangle representing the perimeter of the environment was drawn in scale $(17.92 \times$ $21.18 \mathrm{~cm}$ ). It also specified the southwest corner (i.e., the starting point) of the environment and the direction of the first leg, which was aligned with participants' egocentric orientation at the time of initial presentation of the board. Participants were free to turn the board, if they so chose. They were given 10 identical magnet disks ( $2 \mathrm{~cm}$ diameter), which were to be placed at the locations of landmarks within the rectangle. They were also given names of the 10 landmarks and used them to indicate which disk represented which landmark. They were allowed to place the landmarks in any order and spend unlimited time to complete this task. No error feedback was given to participants.

Following completion of the memory test, participants were presented with a new environment in the same procedure. This study-test sequence was repeated until all four environments were learned and tested. The order of two perspectives was counterbalanced over participants. Four environments were randomly assigned to each perspective with the constraint that each environment appeared in each position of the sequence with equal frequency.

\subsection{DATA ANALYSIS}

The dependent measure was accuracy of the reconstructed layouts of landmarks. It was obtained through extension of bidimensional regression (Tobler, 1994). In this technique, a reconstructed layout first undergoes translation, rotation, and linear scaling so that it is transformed into the correct layout as much as possible. The best-fit layout can be uniquely determined by the least square method, and the regression coefficients associated with this transformation are converted into a distortion index (DI), 
which reflects the overall accuracy of the reconstructed layout independent of translation, rotation, and scaling factors. The DI is a dimensionless value ranging from 0 to 100 , with 0 indicating accurate reconstruction of the layout and 100 corresponding to the case in which all landmarks are placed in a single point. Details of the computational procedure are described by Waterman and Gordon (1984).

Although primary independent variables in this experiment were participant groups (young vs. senior) and types of spatial learning (navigation vs. map), it included an additional variable that was not relevant to the present study: once within each type of learning, the four legs of an environment were presented in random order. However, other than increasing the overall distortion of reconstructed layouts of landmarks, this variable showed virtually no interactions with group or learning type. Thus, this additional variable was not included in the analysis. Dropping this variable did not introduce any bias in results reported below because its effect was equally distributed across all conditions (i.e., the three variables were factorially combined).

\section{RESULTS}

DIs from the two environments learned in the same type of learning were averaged within each participant, and then their means and standard deviations were calculated for each age group and for each learning type. They were as follows (see also Figure 2): $M=47.07, S D=19.63$ (young, navigation); $M=34.27, S D=13.66$ (young, map); $M=65.51, S D=22.02$ (senior, navigation); and $M=35.46, S D=14.27$ (senior, map).

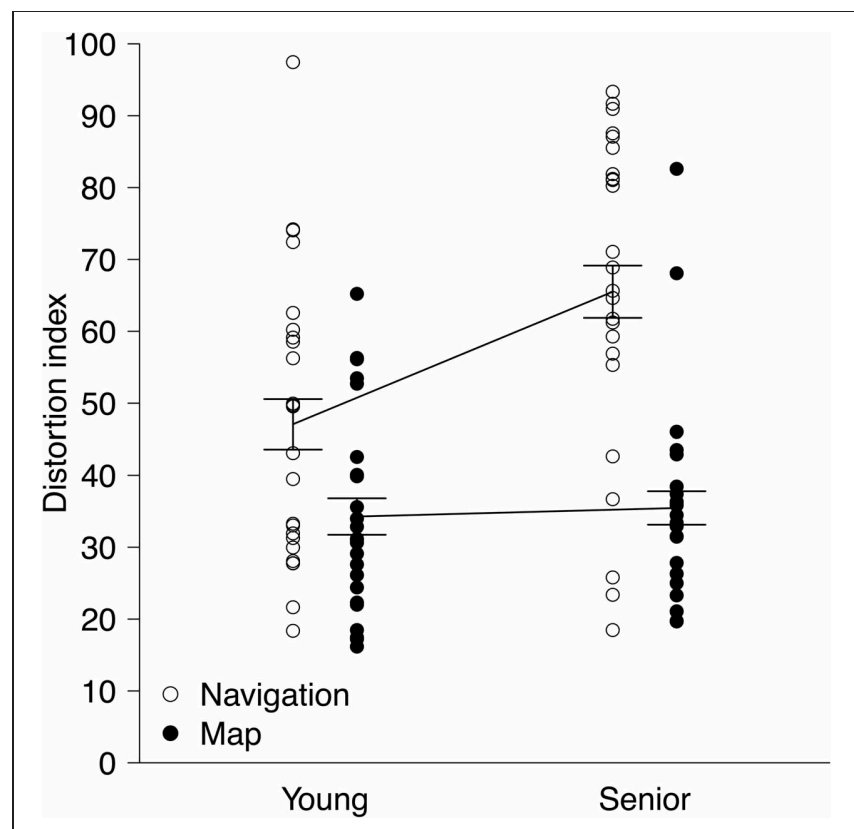

FIGURE 2 | Distortion indices (DIs) as a function of group (young vs. senior) and type of learning (navigation vs. map). Each dot shows a DI of one participant. For each type of learning, a solid line connects the means of the age groups. Error bars represent \pm 1 standard error of the mean.
Data were checked for outliers, but only one data point of a senior participant in the map condition fell outside the range of $M \pm 3$ $S D$, and excluding this participant did not alter the results at all. Thus, all data points were included in the analysis. DIs were subjected to a split-plot analysis of variance with group (young vs. senior) as a between-subject factor and type of learning (navigation vs. map) as a within-subject factor. Generalized eta squared $\left(\eta_{G}^{2}\right)$ values are reported below as effect size statistics (Olejnik and Algina, 2003; Bakeman, 2005).

Figure 2 shows DIs as a function of group and learning type, revealing two major findings. First, following spatial learning through exploratory navigation senior participants created more distorted layouts of landmarks than young participants, but following spatial learning through map reading these two groups performed equivalently. Consistent with these observations, the interaction between group and learning type was significant, $F_{(1,46)}=7.54, p=0.0086, \eta_{\mathrm{G}}^{2}=0.056$. Simple main-effect tests comparing the two age groups yielded a significant result within the navigation condition but not within the map condition, $F_{(1,46)}=12.40, p<0.001, \eta_{\mathrm{G}}^{2}=0.21$, and $F_{(1,46)}=0.052$, $p=0.82, \eta_{\mathrm{G}}^{2}=0.0011$, respectively. Additionally, this substantial worsening of senior participants' performance in the navigation condition made the main effect of group significant, $F_{(1,46)}=5.49, p=0.024, \eta_{\mathrm{G}}^{2}=0.071$. Second, there was an overall difference in the quality of reconstructed layouts between navigation and map conditions: participants reproduced the layouts more accurately after spatial learning through map reading than that through exploratory navigation. This was indicated by the significant main effect of learning type, $F_{(1,46)}=46.56, p<0.001, \eta_{\mathrm{G}}^{2}=0.27$. Furthermore, a simple main-effect test comparing the two conditions in the young group was also significant, $F_{(1,46)}=8.31, p=0.0060, \eta_{\mathrm{G}}^{2}=0.15$, showing that accuracy of reproduced layouts was reliably different between navigation and map conditions even among young participants.

\section{DISCUSSION}

The present study was conducted to investigate whether increasing age has differential impacts on spatial learning through exploratory navigation in the first-person (i.e., ground-level) perspective and map reading in an aerial perspective. On the basis of previous findings that encoding of spatial information from the first-person perspective strongly engages the MTL and that this brain region is susceptible to age-related atrophy, it was hypothesized that elderly adults would be especially impaired at learning an environmental layout by navigating in the environment. Results from the experiment supported this hypothesis, showing that (1) senior participants constructed the layouts of landmarks less accurately than young participants after learning them through exploratory navigation; and (2) young and senior participants reproduced the layouts with equivalent accuracy after learning them through map reading. These data suggest that not all types of spatial learning are affected equally by aging. Rather, it is suggested that seniors' ability in learning environments from maps is better maintained through normal aging processes than their ability to acquire spatial knowledge from actual navigational experiences. 
It is noteworthy that in the experiment young and senior participants performed equivalently after learning environments through maps. This suggests that the observed difference between the two age groups in the navigation condition cannot be explained entirely by general age-related factors such as slower speed of information processing and less familiarity with computers. In navigation and map conditions, the environments were presented at comparable rates (i.e., approximately the same number of landmarks were visible at any given time in both conditions), and identical learning and test procedures were employed. Furthermore, the competent performance of senior participants in the map condition supports the observation that they were cognitively healthy individuals. Thus, it is reasonable to conclude that the decreased accuracy of spatial memories in the navigation condition was indicative of specific effects of normal aging on spatial learning through exploratory navigation.

It is also important to note that although the present study found little difference between young and senior participants in the map condition, it does not necessarily indicate that there would be no age-related decline in map reading abilities. As discussed in the introduction, previous studies showed that elderly adults had a tendency to acquire spatial knowledge from maps less proficiently than young adults (Thomas, 1985; De Beni et al., 2006; Meneghetti et al., 2011). A notable difference from the present study is that in these studies participants were asked to recall both landmark identities and their locations. On the other hand, in the present study, participants were given names of to-be-remembered landmarks in the beginning of a test phase. This most likely reduced the burden on their spatial memories, helping senior participants perform competently in the map condition. Nevertheless, in the navigation condition, this advantage did not eliminate the age difference in spatial learning and memory.

Previous studies on age-related changes in spatial learning suggested that among various processes of spatial learning elderly adults have trouble in cognitive mapping in particular, a process with which observers build the mental representation of a configuration of object locations that are experienced separately (e.g., Iaria et al., 2009; Liu et al., 2011). In these studies, participants learned large-scale environments through exploratory navigation in the first-person perspective and subsequently attempted to create layouts of landmarks from an aerial perspective. Consistent with the navigation condition of the present study, senior participants in the previous studies were impaired at this task compared to young participants. By contrast, although the same cognitive mapping process was involved in the map condition of the present study, senior participants reconstructed the layouts as accurately as young participants. This suggests that the integration process itself with which landmark locations are combined into the entire layout remains intact in elderly adults. Instead, impaired performance observed in the previous studies and in the navigation condition likely stemmed from declines in the ability of (1) encoding spatial information from the ground-level perspective, (2) transforming spatial information from ground-level into aerial perspectives, or (3) aligning landmarks learned from multiple orientations with each other, as none of these was necessary in the map condition.
Related to the above, it should be explicitly pointed out that in the present study the memory test was conducted in an aerial perspective that was also used for encoding spatial information in the map condition. As a consequence, participants had to transform spatial information from ground-level to aerial perspectives only in the navigation condition. This transformation was expected to have minimal influence (at least in young participants) because when a similar task was performed by young adults in previous studies, layouts of equivalent accuracy were constructed after learning environments from ground-level and aerial perspectives (Shelton and Gabrieli, 2002; Shelton and McNamara, 2004; Shelton and Pippitt, 2007). However, in the present study, even young participants created more accurate layouts of landmarks in the map condition than in the navigation condition. Although it is not readily clear why there was this discrepancy in findings, the present result does indicate that effects of perspective transformation were not negligible in the navigation condition. This is potentially important for explaining the observed age-related difference in this condition, given that the MTL has been shown to be involved in manipulation of viewpoints in spatial memory (King et al., 2002; Lee et al., 2005); that is, the ability to mentally transform perspectives can decline in aging, possibly causing selective impairment in the navigation condition (Inagaki et al., 2002; Joanisse et al., 2008; Devlin and Wilson, 2010). Thus, in future studies it would be informative to include tasks that do not require transforming perspectives between learning and test in both navigation and map conditions (e.g., a scene recognition task; Shelton and McNamara, 2004). Such tasks would help clarify whether it was types of spatial learning per se or mental transformation of spatial information that impacted performance of senior participants in the present study.

Spatial learning through exploratory navigation and spatial learning through map reading are different in a number of respects. The present study focused on two most notable differences between them: perspectives and orientations. Exploratory navigation is carried out in a ground-level perspective with variable orientations, whereas maps are read from an aerial perspective in a stable orientation. In the present study, they were varied simultaneously between navigation and map conditions for achieving naturalistic simulation of exploratory navigation and map reading. Although this approach was effective in revealing the selective effect of aging on spatial learning through exploratory navigation, the present data alone cannot determine whether it originated from viewing environments from the ground-level perspective or learning environmental layouts with varying orientations. Similarly, for the purpose of making strict comparisons between the two conditions, some characteristics of each type of spatial learning were not captured in the present study. For example, one typical advantage of map reading over exploratory navigation is that observers can learn a greater portion of an environment from a single point of view. However, this advantage was not available in the present experiment because approximately the same number of landmarks were made visible at any given time in both conditions in order to avoid a potential confound of general age difference in information processing speed. It is important for future studies to examine possible roles 
of these unexplored factors in age-related changes in spatial learning abilities. Such investigations should bring about a clearer understanding of elderly adults' challenges in spatial learning and memory, and eventually a solution for easing their difficulties.

\section{REFERENCES}

Aguirre, G. K., Detre, J. A., Alsop, D. C., and D'Esposito, M. (1996). The parahippocampus subserves topographical learning in man. Cereb. Cortex 6, 823-829.

Antonova, E., Parslow, D., Brammer, M., Dawson, G. R., Jackson, S. H. D., and Morris, R. G. (2009). Agerelated neural activity during allocentric spatial memory. Memory 17, 125-143.

Bakeman, R. (2005). Recommended effect size statistics for repeated measures designs. Behav. Res. Methods 37, 379-384.

Barrash, J. (1994). Age-related decline in route learning ability. Dev. Neuropsychol. 10, 189-201.

Blanch, R. J., Brennan, D., Condon, B., Santosh, C., and Hadley, D. (2004). Are there gender-specific neural substrates of route learning from different perspectives? Cereb. Cortex 14, 1207-1213.

Borghesani, P. R., Johnson, L. C., Shelton, A. L., Peskind, E. R., Aylward, E. H., Schellenberg, G. D., and Cherrier, M. M. (2008). Altered medial temporal lobe responses during visuospatial encoding in healthy $\mathrm{APOE}^{\star} 4$ carriers. Neurobiol. Aging 29, 981-991.

Burns, P. C. (1999). Navigation and the mobility of older drivers. J. Gerontol. B Psychol. Sci. Soc. Sci. 54B, S49-S55.

Carelli, L., Rusconi, M. L., Scarabelli, C., Stampatori, C., Mattioli, F., and Riva, G. (2011). The transfer from survey (map-like) to route representations into virtual reality mazes: effect of age and cerebral lesion. J. Neuroeng. Rehabil. 8, 6.

Cohen, J., MacWhinney, B., Flatt, M., and Provost, J. (1993). PsyScope: an interactive graphic system for designing and controlling experiments in the psychology laboratory using Macintosh computers. Behav. Res. Methods Instrum. Comput. 25, 257-271.

Cushman, L. A., Stein, K., and Duffy, C. J. (2008). Detecting navigational deficits in cognitive aging and Alzheimer disease using virtual reality. Neurology 71, 888-895.

De Beni, R., Pazzaglia, F., and Gardini, S. (2006). The role of mental rotation and age in spatial perspective-taking tasks: when age does not impair perspective-taking performance. Appl. Cogn. Psychol. 20, 807-821.

Devlin, A. L., and Wilson, P. H. (2010). Adult age differences in the ability to mentally transform object and body stimuli. Aging Neuropsychol. Cogn. 17, 709-729.

Golomb, J., de Leon, M. J., Kluger, A., George, A. E., Tarshish, C., and Ferris, S. H. (1993). Hippocampal atrophy in normal aging. Arch. Neurol. 50, 967-973.

Grön, G., Wunderlich, A. P., Spitzer, M., Tomczak, R., and Riepe, M. W. (2000). Brain activation during human navigation: gender-different neural networks as substrate of performance. Nat. Neurosci. 3, 404-408.

Iachini, T., Iavarone, A., Senese, V. P., Ruotolo, F., and Ruggiero, G. (2009). Visuospatial memory in healthy elderly, $\mathrm{AD}$ and MCI: a review. Curr. Aging Sci. 2, 43-59.

Iachini, T., Poderico, C., Ruggiero, G., and Iavarone, A. (2005). Age differences in mental scanning of locomotor maps. Disabil. Rehabil. 27, 741-752.

Iaria, G., Palermo, L., Committeri, G., and Barton, J. J. S. (2009). Age differences in the formation and use of cognitive maps. Behav. Brain Res. 196, 187-191.

Iglói, K., Doeller, C. F., Berthoz, A., Rondi-Reig, L., and Burgess, N. (2010). Lateralized human hippocampal activity predicts navigation based on sequence or place memory. Proc. Natl. Acad. Sci. U.S.A. 107, 14466-14471.

Inagaki, H., Meguro, K., Shimada, M., Ishizaki, J., Okuzumi, H., and between mental rotation and perspective-taking abilities in normal aging assessed by Piaget's three-mountain task. J. Clin. Exp. Neuropsychol. 24, 18-25.

Insausti, R., Insausti, A. M., Sobreviela, M. T., Salinas, A., and MartínezPeñuela, J. M. (1998). Human medial temporal lobe in aging: anatomical basis of memory preservation. Microsc. Res. Tech. 43, 8-15.

Jack, C. R., Petersen, R. C., Xu, Y., O’Brien, P. C., Smith, G. E., Ivnik, R. J., Tangalos, E. G., and Kokmen, E. (1998). The rate of medial temporal lobe atrophy in typical aging Yamadori, A. (2002). Discrepancy

\section{ACKNOWLEDGMENTS}

The authors thank Amy Shelton for providing materials used in the experiment, and Albert Smith, Jesse Sargent, and Lucinda Rohde for comments on drafts of this article.

and Alzheimer's disease. Neurology 51, 993-999.

Jack, C. R., Petersen, R. C., Xu, Y C., Waring, S. C., O'Brien, P. C. Tangalos, E. G., Smith, G. E., Ivnik, R. J., and Kokmen, E. (1997). Medial temporal atrophy on MRI in normal aging and very mild Alzheimer's disease. Neurology 49, 786-794.

Jernigan, T. L., Archibald, S. L. Fennema-Notestine, C., Gamst, A. C., Stout, J. C., Bonner, J., and Hesselink, J. R. (2001). Effects of age on tissues and regions of the cerebrum and cerebellum. Neurobiol. Aging 22, 581-594.

Joanisse, M., Gagnon, S., Kreller, J., and Charbonneau, M.-C. (2008). Age-related differences in viewer-rotation tasks: is mental manipulation the key factor? J. Gerontol. B Psychol. Sci. Soc. Sci. 63B, P193-P200.

King, J. A., Burgess, N., Hartley, T. Vargha-Khadem, F., and O'Keefe, J. (2002). Human hippocampus and viewpoint dependence in spatial memory. Hippocampus 12 811-820.

Kirasic, K. C. (1991). Spatial cognition and behavior in young and elderly adults: implications for learning new environments. Psychol. Aging 6 , 10-18.

Lee, A. C. H., Buckley, M. J., Pegman, S J., Spiers, H., Scahill, V. L., Gaffan, D., Bussey, T. J., Davies, R. R., Kapur, N., Hodges, J. R., and Graham, K. S. (2005). Specialization in the medial temporal lobe for processing of objects and scenes. Hippocampus $15,782-797$.

Liu, I., Levy, R. M., Barton, J. J. S. and Iaria, G. (2011). Age and gender differences in various topographical orientation strategies. Brain Res. $1410,112-119$.

Lövdén, M., Schellenbach, M. Grossman-Hutter, B., Krüger A., and Lindenberger, U. (2005) Environmental topography and postural control demands shape aging-associated decrements in spatial navigation performance. Psychol. Aging 20, 683-694.

Marchette, S. A., Bakker, A., and Shelton, A. L. (2011). Cognitive mappers to creatures of habit: differential engagement of place and response learning mechanisms predicts human navigational behavior. J. Neurosci. 31, 15264-15268.
Meneghetti, C., Fiore, F., Borella, E., and De Beni, R. (2011). Learning a map of environment: the role of visuo-spatial abilities in young and older adults. Appl. Cogn. Psychol. 25, 952-959.

Meulenbroek, O., Petersson, K. M., Voermans, N., Weber, B., and Fernández, G. (2004). Age differences in neural correlates of route encoding and route recognition. Neuroimage 22, 1503-1514.

Moffat, S. D. (2009). Aging and spatial navigation: what do we know and where do we go? Neuropsychol. Rev. 19, 478-489.

Moffat, S. D., Elkins, W., and Resnick, S. M. (2006). Age differences in the neural systems supporting human allocentric spatial navigation. Neurobiol. Aging 27, 965-972.

Moffat, S. D., and Resnick, S. M. (2002). Effects of age on virtual environment place navigation and allocentric cognitive mapping. Behav. Neurosci. 116, 851-859.

Moffat, S. D., Zonderman, A. B., and Resnick, S. M. (2001). Age differences in spatial memory in a virtual environment navigation task. Neurobiol. Aging 22, 787-796.

Nedelska, Z., Andel, R., Laczó, J., Vlcek, K., Horinek, D., Lisy, J., Sheardova, K., Bureš, J., and Hort, J. (2012). Spatial navigation impairment is proportional to right hippocampal volume. Proc. Natl. Acad. Sci. U.S.A. 109, 2590-2594.

Newman, M. C., and Kaszniak, A. W. (2000). Spatial memory and aging: performance on a human analog of the Morris water maze. Aging Neuropsychol. Cogn. 7, 86-93.

Olejnik, S., and Algina, J. (2003). Generalized eta and omega squared statistics: measures of effect size for some common research designs. Psychol. Methods 8, 434-447.

Pai, M.-C., and Jacobs, W. J. (2004). Topographical disorientation in community-residing patients with Alzheimer's disease. Int. J. Geriatr. Psychiatry 19, 250-255.

Raz, N., Gunning-Dixon, F., Head, D., Rodrigue, K. M., Williamson, A., and Acker, J. D. (2004). Aging, sexual dimorphism, and hemispheric asymmetry of the cerebral cortex: replicability of regional differences 
in volume. Neurobiol. Aging 25, 377-396.

Scahill, R. I., Frost, C., Jenkins, R., Whitwell, J. L., Rossor, M. N., and Fox, N. C. (2003). A longitudinal study of brain volume changes in normal aging using serial registered magnetic resonance imaging. Arch. Neurol. 60, 989-994.

Shelton, A. L., and Gabrieli, J. D. E. (2002). Neural correlates of encoding space from route and survey perspectives. J. Neurosci. 22, 2711-2717.

Shelton, A. L., and McNamara, T. P. (2004). Orientation and perspective dependence in route and survey learning. J. Exp. Psychol. Learn. Mem. Cogn. 30, 158-170.

Shelton, A. L., and Pippitt, H. A. (2007). Fixed versus dynamic orientations in environmental learning from ground-level and aerial perspectives. Psychol. Res. 71, 333-346.

Siegel, A. W., and White, S. H. (1975).

"The development of spatial representations of large-scale environments," in Advances in Child Development and Behavior, ed $\mathrm{H}$. W. Reese (New York, NY: Academic Press), 9-55.

Šimić, G., Kostović, I., Winblad, B., and Bogdanović, N. (1997). Volume and number of neurons of the human hippocampal formation in normal aging and Alzheimer's disease. J. Comp. Neurol. 379, 482-494.

Sjölinder, M., Höök, K., Nilsson, L.-G., and Andersson, G. (2005). Age differences and the acquisition of spatial knowledge in a three-dimensional environment: evaluating the use of an overview map as a navigation aid. Int. J. Hum. Comput. Stud. 63, 537-564.

Strittmatter, W. J., Saunders, A. M., Schmechel, D., Pericak-Vance, M., Enghild, J., Salvesen, G. S., and Roses, A. D. (1993). Apolipoprotein E: High-avidity binding to $\beta$ amyloid and increased frequency of type 4 allele in late-onset familial Alzheimer disease. Proc. Natl. Acad. Sci. U.S.A. 90, 1977-1981.

Sullivan, E. V., March, L., Mathalon, D. H., Lim, K. O., and Pfefferbaum, A. (1995). Age-related decline in MRI volumes of temporal lobe gray matter but not hippocampus. Neurobiol. Aging 16, 591-606.

Sullivan, E. V., Marsh, L., and Pfefferbaum, A. (2005). Preservation of hippocampal volume throughout adulthood in healthy men and women. Neurobiol. Aging 26, 1093-1098.

Thomas, J. L. (1985). Visual memory: adult age differences in map recall and learning strategies. Exp. Aging Res. 11, 93-95.

Thorndyke, P. W., and Hayes-Roth, B. (1982). Differences in spatial knowledge acquired from maps and navigation. Cogn. Psychol. 14, 560-589.

Tobler, W. R. (1994). Bidimensional regression. Geogr. Anal. 26, 187-212.

Vlček, K. (2011). "Spatial navigation impairment in healthy aging and Alzheimer's disease," in The Clinical Spectrum of Alzheimer's Disease: The Charge Toward Comprehensive Diagnostic and Therapeutic Strategies, ed S. M. de la Monte (Rijeka, Croatia: InTech), 75-100.

Waterman, S., and Gordon, D. (1984). A quantitative-comparative approach to analysis of distortion in mental maps. Prof. Geogr. 36, 326-337.

Wilkniss, S. M., Jones, M. G., Korol, D. L., Gold, P. E., and Manning, C. A. (1997). Age-related differences in an ecologically based study of route learning. Psychol. Aging 12, 372-375.
Zakzanis, K. K., Quintin, G., Graham, S. J., and Mraz, R. (2009). Age and dementia related differences in spatial navigation within an immersive virtual environment. Med. Sci. Monit. 15, CR140-CR150.

Conflict of Interest Statement: The authors declare that the research reported in this article was conducted in the absence of any commercial or financial relationships that could be construed as a potential conflict of interest.

Received: 27 March 2012; accepted: 29 May 2012; published online: 12 June 2012.

Citation: Yamamoto $N$ and DeGirolamo GJ (2012) Differential effects of aging on spatial learning through exploratory navigation and map reading. Front. Ag. Neurosci. 4:14. doi: 10.3389/fnagi. 2012.00014

Copyright (C) 2012 Yamamoto and DeGirolamo. This is an open-access article distributed under the terms of the Creative Commons Attribution Non Commercial License, which permits noncommercial use, distribution, and reproduction in other forums, provided the original authors and source are credited. 